Bulletin of Mathematical Biology (1999) 61, 1093-1120

Article No. bulm.1999.0131

Available online at http://www.idealibrary.com on IDE $\mathbf{A}$

\title{
Reaction and Diffusion on Growing Domains: Scenarios for Robust Pattern Formation
}

\author{
EDMUND J. CRAMPIN*, EAMONN A. GAFFNEY AND PHILIP K. \\ MAINI \\ Centre for Mathematical Biology, \\ Mathematical Institute, \\ 24-29 St Giles', Oxford, OX1 3LB, U.K. \\ E-mail: crampin@maths.ox.ac.uk
}

\begin{abstract}
We investigate the sequence of patterns generated by a reaction-diffusion system on a growing domain. We derive a general evolution equation to incorporate domain growth in reaction-diffusion models and consider the case of slow and isotropic domain growth in one spatial dimension. We use a self-similarity argument to predict a frequency-doubling sequence of patterns for exponential domain growth and we find numerically that frequency-doubling is realized for a finite range of exponential growth rate. We consider pattern formation under different forms for the growth and show that in one dimension domain growth may be a mechanism for increased robustness of pattern formation.
\end{abstract}

(C) 1999 Society for Mathematical Biology

\section{INTRODUCTION}

The reaction-diffusion mechanism is one of the simplest and most elegant pattern formation models. Turing (1952) first proposed the mechanism in the context of biological morphogenesis, showing that reactions between two diffusible chemicals (morphogens) could give rise to spatially heterogeneous concentrations through an instability driven by diffusion. He further suggested that morphogen concentration patterns formed in this manner might be the source of spatial organization in the embryo; developmental fate subsequently being cued by cellular mechanisms in response to thresholds in morphogen concentration. In this, as in most pattern formation applications of reaction-diffusion systems, it is supposed that the diffusion and reaction take place on a timescale that is much faster than the mechanism interpreting the spatial information. The establishment and the interpretation of the spatial pattern then decouple allowing pattern formation to be studied independently and for this reason many theoretical studies have considered only the existence and stability of patterned steady state solutions.

It has been suggested (Oster and Murray, 1989) that other lateral inhibition models for global pattern formation, in which the entire domain is patterned simultaneously, have equivalent underlying mathematical structure and differ significantly

\footnotetext{
*Author to whom correspondence should be addressed.

$0092-8240 / 99 / 061093+28 \quad \$ 30.00 / 0$

(c) 1999 Society for Mathematical Biology
} 
only in their biological interpretation. Accordingly, one might usefully study the behaviour of the equations underlying such models out of the context of the biological motivation for any particular model. In this paper we use reaction-diffusion theory as a paradigm within which to study the qualitative effects of domain growth on pattern formation. We suppose that the domain is growing on a timescale commensurate with pattern generation such that it may not be decoupled from the reactiondiffusion mechanism. We consider the generation of a sequence of patterns and discuss the dynamics and transient behaviour responsible for the sequence. We find that domain growth imposes a constraint on the system which restricts the set of accessible patterns, increasing the robustness of pattern generation with respect to the initial conditions. The improvement in robustness of pattern selection is the key feature of the model that we present, which has clear consequences for the applications of global pattern formation models.

1.1. Pattern selection and robustness. Reaction-diffusion models may admit multiple heterogeneous solutions for a given parameter set, with pattern selection sensitive to initial conditions (Arcuri and Murray, 1986), spatial scale and geometry. Precise control of the initial conditions and equation parameters is required to ensure the selection of a specific pattern. This is a generic feature of global pattern generators. Applications of reaction-diffusion models in various contexts have been criticised for a lack of robustness (Bard and Lauder, 1974; Bunow et al., 1980). Indeed, some authors have concluded that global pattern formation mechanisms alone cannot be the source of reliable spatial pattern under normal biological variation. In a model for segmentation, where the major focus is on the reliable generation of stripes, Lacalli et al. (1988) introduced a spatial gradient in one of the model parameters along the axis of stripe formation in order to obtain a large number of stripes (for example 8) reliably. Murray (1982) has compared pattern formation under various kinetic systems and has ranked models in terms of the relative sizes of parameter space regions for pattern formation (the so-called Turing space). In this paper we are concerned with pattern sensitivity to the size of the domain. The reliable establishment of pattern is found to be particularly difficult for larger solution domains (which admit higher pattern modes) and for systems in two and three spatial dimensions, where sensitivity to initial conditions and geometry becomes increasingly pronounced.

We note that this sensitivity is a positive asset to some models incorporating a reaction-diffusion mechanism, for example in models of animal coat markings (Murray, 1981), where different members of the same species display patterns which are characterized by a similar wavelength but which in detail show great variety between animals. This is also a feature of similar models for ocular dominance columns (Swindale, 1980) and finger prints (Bentil and Murray, 1992) where some amount of variability in pattern is desirable. 
1.2. Growth. Previously, several authors have incorporated domain growth into models of pattern formation, usually by introducing or modifying terms in an ad hoc manner. Arcuri and Murray (1986) have proposed a caricature model in which a nondimensional parameter containing the domain length is assigned an explicit time dependence. The results of numerical simulations reported in their paper show a tendency for the omission of modes in the sequences of patterns generated by the model, and under certain (unclear) circumstances a tendency to frequencydoubling was also noted. Here we develop a similar model from first principles, and find that the frequency-doubling sequence is a robust and pervasive solution behaviour.

Kulesa et al. (1996) have incorporated exponential domain growth into a model for the spatio-temporal sequence of initiation of tooth primordia in the Alligator mississippiensis. In the model domain growth plays a central role in establishing the order in which tooth precursors appear. Recently, stemming from work by Kondo and Asai (1995), various authors have considered the effect of growth in numerical simulations of reaction-diffusion models for the skin patterns on species of fish during their development from juvenile to adult forms (Varea et al., 1997; Painter et al., 1999). Here the pattern change is commensurate with growth and shape change, and many different pattern behaviours are observed in response to the growing domain before the final domain geometry is achieved. This is a particularly exciting experimental system in which pattern and shape change are easily observed and quantified over time, and lends strong circumstantial evidence that a global reaction-diffusion-type mechanism may be responsible for the evolving patterns. In a paper considering models for reliable segmentation Saunders and Ho (1995) propose hierarchical division of a domain by setting up a sequence of internal boundaries. The authors consider pattern formation in one spatial dimension, dismissing the reaction-diffusion mechanism because of the lack of scale invariance on the fixed domain and also by the apparent failure of the mechanism to generate a reliable pattern sequence with domain growth, based on the results of Arcuri and Murray. They report that the inclusion of growth contributes another source of instability, that such a mechanism cannot produce a reliable 16 segment pattern, and conclude that they would not expect any mechanism that acts over the whole domain to be capable of robust pattern formation at higher modes (p. 547). The results that we present here suggest, at least in one-dimension, that growth may in fact stabilize the frequency-doubling sequence and subsequently that it may be a mechanism for robust pattern formation.

In the following section we briefly describe standard reaction-diffusion theory on the fixed domain, highlighting those results which are pertinent to the present study. In Section 3 we derive evolution equations for reaction-diffusion on a generalized growing domain and describe a model for slow and isotropic growth, which will be the subject of the remainder of this paper. In Section 4 we describe the frequency-doubling sequence and use a notion of self-similarity to predict growth functions under which this pattern sequence is realized. We provide numerical evi- 
dence in support of our analysis and examine pattern generation under other growth functions. Finally we discuss our results with reference to the topics raised in this introduction.

\section{REACTION-DIFFUSION THEORY}

First we define a reaction-diffusion equation describing the interaction of $n$ chemical species, denoted by the concentration vector $c=\left(c_{1}, c_{2}, \ldots, c_{n}\right)$, on a domain $\Omega$ in $\mathbb{R}^{N}$. We consider below the general case for a rectangular domain of dimension $N \leq 3$, although we will concentrate on the one-dimensional problem for much of the remainder of this paper. Ordering the chemicals with decreasing diffusivity and transforming spatial coordinates to the unit interval we have the nondimensional evolution equation

$$
\frac{\partial c}{\partial t}=\frac{1}{\gamma} \mathcal{D} \nabla^{2} c+R(c), \quad \mathbf{x} \in[0,1]^{N}
$$

describing the spatio-temporal distribution of chemicals with initial conditions $c(\mathbf{x}, 0)$. Here $\nabla^{2}$ is the $N$-dimensional Laplacian and $\mathcal{D}=\operatorname{diag}\left\{1, d_{2}, \ldots, d_{n}\right\}$ is the diagonal matrix of diffusivities with no cross-diffusion. The diffusivities are given relative to the largest, $D_{1}$, such that $d_{i}=D_{i} / D_{1} \leq 1$ and $\gamma$, the dimensionless scaling parameter, is given by $\gamma=\omega L^{2} / D_{1}$. The timescale $\omega$ is characteristic of the reaction kinetics vector $R$, and $L$ is a length scale usually taken to be the (initial) domain length for problems in one spatial dimension. The parameter $\gamma$, scaling the diffusion terms, determines the relative strengths of interaction of the reaction and diffusion terms. Solutions are influenced by the domain size through $\gamma$. To close the problem we impose boundary conditions on the solution. A natural choice is to assume that the boundaries do not influence the interior of the domain and impose zero flux (Neumann) conditions. We will comment on this choice later with reference to the role of boundary conditions in pattern formation.

2.1. Diffusion-driven instability. In his paper of 1952 Turing showed that such a reaction-diffusion system with two chemicals, $n=2$, may generate spontaneous, symmetry-breaking spatial pattern. This fundamental idea has since been explored and developed in numerous texts. One of the novel features of the mechanism is that the length scale of the pattern is inherent to the mechanism, rather than imposed by the size of the solution domain as is the case for other pattern formation models. Recently interest has been revived by the first experimental realisation of stationary patterns in a chemical reaction [see Castets et al. (1990); De Kepper et al. (1991) and Maini et al. (1997) for a recent review].

Generally, two-component schemes capable of pattern formation include selfactivation with short-range signalling and self-inhibition with longer range signalling. In chemical terms such systems comprise either a short-range self-activator 
which activates and is reciprocally inhibited by a long-range self-inhibitor (pure kinetics), or a self-activator that depletes the self-inhibitor (substrate) which in turn feeds production of the activator (cross kinetics). The necessary conditions for bifurcation from a homogeneous spatial distribution to a patterned state can be determined from the diffusivities of the chemicals and the kinetic parameters in the linearized model [Dillon et al. (1994), for example]. For $d=D_{2} / D_{1} \leq 1$, linear analysis determines critical parameter values $d_{c}$ and $\gamma_{c}$ such that we require $d<d_{c}$ and $\gamma>\gamma_{c}$ for diffusion-driven instability, along with conditions on the linear part of the kinetics. The condition on $\gamma$ determines a minimum domain length: we require that the domain admit at least one half-cycle of the intrinsic pattern wavelength. The analysis predicts that the homogeneous steady state is destabilized by sinusoidal modes, with amplitude growing exponentially, and that an increasing number of modes are able to destabilize the homogeneous state with increasing $\gamma$. Away from the bifurcation point, weakly nonlinear analysis can be used to investigate the modification of the pattern in the nonlinear regime. We will refer to different patterns by the appropriate linear mode number, $m$. The Turing bifurcation has pitchfork structure and so a pattern based on mode $m$ may take either of two polarities, $\pm \cos m \pi x$. Activator and inhibitor patterns are in phase for a pure kinetic system while for cross systems the spatial oscillations are in antiphase. The polarity adopted by the solution in general depends on the initial conditions, as does selection of the solution branch and hence pattern mode. The increasing multiplicity of solution branches for increasing $\gamma$ implies increasing sensitivity to the initial conditions for increasing domain length.

2.2. Boundary and initial conditions: sensitivity of pattern selection. General boundary conditions are written as

$$
\mathbf{n} \cdot \nabla c=\mathcal{H}\left(c^{*}-c\right), \quad \mathbf{x} \in \partial \Omega
$$

where $c^{*}$ is a fixed reference concentration vector. $\mathcal{H}$ is the nondimensional mass transfer matrix which, excluding cross-species dependency, is given by $\mathcal{H}=$ diag $\left\{h_{1}, h_{2}, \ldots, h_{n}\right\}$. When $h_{i}=h$ for all $i$ we have scalar boundary conditions, of which the special cases of zero flux (Neumann) and homogeneous Dirichlet conditions are given by $h=0$ and $\infty$, respectively. More general combinations of Dirichlet and Neumann conditions in the different species are known as nonscalar (mixed) conditions. Homogeneous conditions are those for which $c^{*}=c^{s}$, where $R\left(c^{s}\right)=0$ defines the kinetic steady state for which a spatially homogeneous steady state solution exists and is stable below a critical domain size.

Arcuri and Murray (1986) have studied numerically the influence of initial conditions on the pattern generation of two-species reaction-diffusion equations in one spatial dimension as a function of the imposed boundary conditions. The sensitivity of the pattern selection to the initial data on the domain was found to decrease with increasing inhomogeneity of the boundary conditions, i.e., as $\left|c^{*}-c^{s}\right|$ increases. 


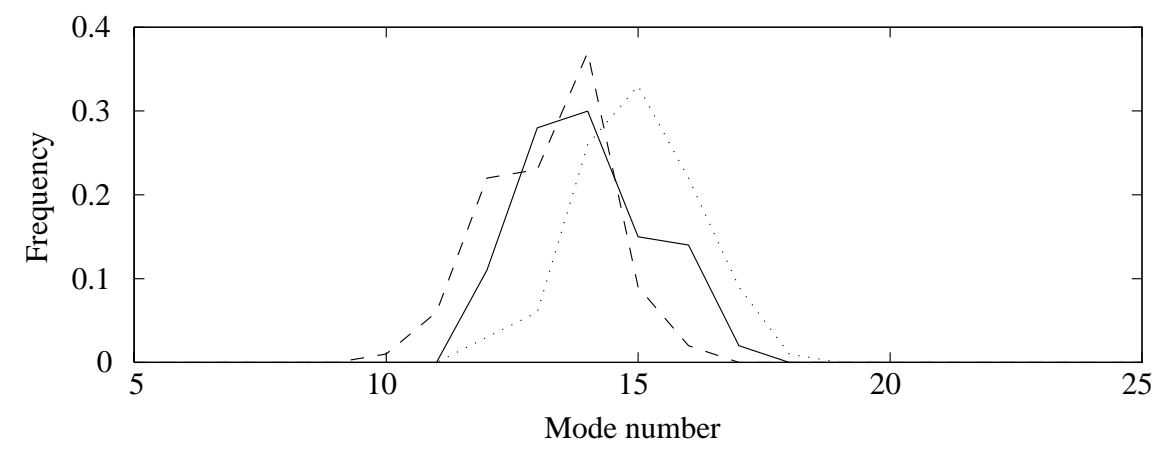

Figure 1. Relative frequencies of steady state solution modes against mode number $m$ for numerical simulation of a reaction-diffusion equation (1) with Schnakenberg kinetics (24) for three different values of $\gamma$. Each simulation took different random initial conditions with zero flux boundary conditions. Dashed, solid and dotted lines correspond to $\gamma=190,215$ and 240 for which the fastest growing linear mode is $m=15,16$ and 17, respectively.

Dillon et al. (1994) have studied the steady state solutions of reaction-diffusion systems under nonscalar boundary conditions using numerical bifurcation techniques. The multiplicity of stable pattern solutions characteristic of the scalar case and the sensitivity to initial data can be shown to be reduced under such conditions. Using the domain length as the bifurcation parameter, the authors showed that the bifurcation diagram is simplified, signifying a reduction in sensitivity of pattern selection to the domain length. Also the constraint on the ratio of diffusivities for diffusion-driven instability is somewhat relaxed.

It is apparent from these studies that zero flux boundary conditions impose the weakest constraint on pattern formation and lead to the greatest difficulty in achieving reliable pattern selection from initial conditions. We will assume these boundary conditions, the worst case scenario, for our investigation of patterning during growth of the domain. To illustrate the lack of reliable pattern selection we show in Fig. 1 the relative frequencies of various steady state pattern modes developing from different sets of random initial conditions on a domain of fixed length $L$, for various values of $L$. The three domain lengths chosen correspond to values of $L$ for which the linear stability analysis predicts three sequential modes to be fastest growing in the linear regime. Similar studies at different domain length show increasing variance of the distribution of mode selected with increasing fixed domain length.

2.3. Steady state patterns in one dimension. In this section we construct new solutions for the steady state equations from low-mode solutions on a fixed domain. The method that we employ here illustrates the self-similarity property which we 
will exploit later to give insight into the growing domain problem (see Section 4). We consider solutions to the steady state problem

$$
0=\frac{1}{\gamma} \mathcal{D} \frac{\mathrm{d}^{2} c}{\mathrm{~d} x^{2}}+R(c), \quad x \in[0,1]
$$

with zero flux boundary conditions

$$
\frac{\mathrm{d} c}{\mathrm{~d} x}=0, \quad x \in\{0,1\} .
$$

Let us suppose that at $\gamma=\gamma_{1}>\gamma_{c}$ the solution consists of the fundamental mode $m=1$, where the linearized equations have solution with heterogeneity $\cos \pi x$. We can construct new solutions by scaling, translating and reflecting this pattern. To obtain a new pattern, $q_{2}(x)$, of mode 2 and of the same polarity we use the tent map transformation

$$
p_{2}(x)= \begin{cases}2 x & 0 \leq x<\frac{1}{2} \\ 2-2 x & \frac{1}{2} \leq x \leq 1\end{cases}
$$

such that

$$
q_{2}\left(x ; \gamma_{1}\right) \equiv c\left(p_{2}(x) ; \gamma_{1}\right)
$$

which satisfies the equation

$$
0=\frac{1}{4 \gamma_{1}} \mathcal{D} \frac{\mathrm{d}^{2} q_{2}}{\mathrm{~d} x^{2}}+R\left(q_{2}\right) .
$$

The transformation $p_{2}(x)$ ensures that the zero flux conditions are satisfied at the boundaries of the unit interval, and so $q_{2}(x ; \gamma)$ is a solution of the same equation as $c\left(x ; \gamma_{1}\right)$ when $\gamma=4 \gamma_{1}$. The transformation has discontinuous derivative at the point $x=\frac{1}{2}$, however, $q_{2}(x)$ has zero gradient here by construction and therefore is twice continuously differentiable. Under the transformation $p_{2}(x)$ we have chosen to maintain the parity of the original pattern mode. Patterns with the opposite polarity are similarly constructed by the complementary transformation

$$
\bar{p}_{2}(x)= \begin{cases}1-2 x & 0 \leq x<\frac{1}{2} \\ 2 x-1 & \frac{1}{2} \leq x \leq 1 .\end{cases}
$$

It is straightforward to show that a general transformation $p_{m}(x)$ can be defined in a similar manner to generate a pattern of mode $m$ on the interval $[0,1]$ and to find the corresponding equation. In general $q_{m}(x ; \gamma) \equiv c\left(p_{m}(x) ; \gamma\right)$ satisfies the same equation as $c\left(x ; \gamma_{1}\right)$ when $\gamma=m^{2} \gamma_{1}$. 


\section{REACTION AND Diffusion ON GROWING Domains}

In this section we derive the evolution equations describing the interaction of $n$ chemical species reacting in and diffusing through a growing domain $\Omega_{t}$. The conservation equation in integral form is given by

$$
\frac{\mathrm{d}}{\mathrm{d} t} \int_{\Omega_{t}} c(\mathbf{x}, t) \mathrm{d} \mathbf{x}=\int_{\Omega_{t}}[-\nabla \cdot \mathbf{j}+R(c)] \mathrm{d} \mathbf{x}
$$

where $\mathbf{j}$ is the flux. We use the Reynolds transport theorem to evaluate the left-hand side:

$$
\frac{\mathrm{d}}{\mathrm{d} t} \int_{\Omega_{t}} c(\mathbf{x}, t) \mathrm{d} \mathbf{x}=\int_{\Omega_{t}}\left[\frac{\partial c}{\partial t}+\nabla \cdot \mathbf{u} c\right] \mathrm{d} \mathbf{x}
$$

where $\mathbf{u}(\mathbf{x}, t)$ is the flow and, nondimensionalizing, we recover the evolution equation

$$
\frac{\partial c}{\partial t}+\nabla \cdot(\mathbf{u} c)=\frac{D_{1}}{\omega L^{2}} \mathcal{D} \nabla^{2} c+R(c)
$$

The growing domain, $\Omega_{t}$, introduces an advection term, $\mathbf{u} \cdot \nabla c$, corresponding to elemental volumes moving with the flow due to local growth and a dilution term, $c \nabla \cdot \mathbf{u}$, due to local volume change. While the flow might be determined by specifying the local rate of volumetric growth, perhaps determined by some other tissue process, we chose to define a growth function, $\Gamma$, directly using the Lagrangian description

$$
\mathbf{x}=\Gamma(\mathbf{X}, t), \quad \mathbf{x} \in \Omega_{t}
$$

where $\mathbf{X}$ is an initial position marker. A restriction on $\Gamma$ is that $\Gamma(\mathbf{X}, 0)=\mathbf{X}$. The inverse at time $t$ is given by $\Lambda$ where

$$
\mathbf{X}=\Lambda(\mathbf{x}, t), \quad \mathbf{X} \in \Omega_{0}
$$

where $\Omega_{0}$ is initial domain. The local flow is then fully determined, and is given by

$$
\mathbf{u}(\mathbf{x}, t)=\frac{\partial \mathbf{x}}{\partial t}=\frac{\partial \Gamma}{\partial t}
$$

where the partial derivatives are evaluated at constant $\mathbf{X}$. We have taken no account of mechanical properties of the tissue, only that it is growing due to local volumetric increase. 
3.1. Slow isotropic growth in one spatial dimension. This formulation allows the incorporation of any spatio-temporal growth behaviour in the tissue, which may be determined by some underlying pattern or controlled dynamically by the chemical concentrations themselves. This proposition, reactant-controlled growth, is currently under investigation (Crampin et al., 1999). In the present paper we will restrict our discussion to the simple case of one spatial dimension, and consider isotropic growth given by

$$
\Gamma(X, t)=X r(t), \quad r(0)=1 .
$$

Then the flow is determined by

$$
u(x, t)=X \dot{r}=x \frac{\dot{r}}{r}
$$

after which (11) becomes

$$
\frac{\partial c}{\partial t}+\frac{\dot{r}}{r}\left[x \frac{\partial c}{\partial x}+c\right]=\frac{D_{1}}{\omega L^{2}} \mathcal{D} \frac{\partial^{2} c}{\partial x^{2}}+R(c) .
$$

A natural choice is to transform to the unit interval:

$$
(x, t) \rightarrow(\bar{x}, \bar{t})=\left(\frac{x}{L(t)}, t\right),
$$

where domain length $L(t)=L_{0} r(t)$. We note in particular that

$$
\frac{\partial c}{\partial \bar{t}}=\frac{\partial c}{\partial t}+x \frac{\dot{r}}{r} \frac{\partial c}{\partial x}
$$

and so the transformation eliminates the advection term, reflecting the isotropic transport through the domain ( $r$ is independent of position). On dropping the overbars we recover

$$
\frac{\partial c}{\partial t}=\frac{1}{\omega(L(t))^{2}} \mathcal{D} \frac{\partial^{2} c}{\partial x^{2}}+R(c)-c \frac{\dot{r}}{r} .
$$

We define the time-dependent scaling parameter

$$
\gamma(t) \equiv \frac{\omega L_{0}^{2}}{D_{1}} r^{2}(t)=\gamma_{0} r^{2}(t)
$$

for initial domain length $L_{0}$.

For slow growth $\dot{r}$ is small, and in particular $c \dot{r} / r$ is much smaller than linear terms in $R$ and so we neglect the dilution term to simplify the following analysis. We note that the analysis below can be carried through mutatis mutandis and 
the results are unaffected if the term is included. We obtain the nonautonomous reaction-diffusion system

$$
\begin{aligned}
\frac{\partial c}{\partial t} & =\frac{1}{\gamma} \mathcal{D} \frac{\partial^{2} c}{\partial x^{2}}+R(c), \quad x \in[0,1] \\
\frac{\mathrm{d} \gamma}{\mathrm{d} t} & =h(t)
\end{aligned}
$$

where the second equation defines $h(t)$, and we have zero flux boundary conditions for $c$ and initial conditions $c(x, 0)$ and $\gamma(0)=\gamma_{0}$. These equations generate a sequence of patterns in time. For slow growth, writing $\mathrm{d} \gamma / \mathrm{d} t \sim \varepsilon \ll 1$, we can identify two dynamic regimes:

- If $\partial c / \partial t \ll(1 / \varepsilon)$ then introducing a slow timescale, $\tau=\varepsilon t$, reduces the evolution to a quasi-steady state problem (the solution profile is slowly modulated as $\gamma$ varies).

- However, when $\partial c / \partial t \sim \mathcal{O}(1 / \varepsilon)$ the quasi-stationarity is lost, corresponding to fast transitions between quasi-steady patterns.

We illustrate this with a concrete example for $c=\left(c_{1}, c_{2}\right)$, using the two-component Schnakenberg kinetic scheme (Schnakenberg, 1979) (where $c_{2}$ is the activator):

$$
R=\left(\begin{array}{c}
b-c_{1} c_{2}^{2} \\
a-c_{2}+c_{1} c_{2}^{2}
\end{array}\right)
$$

Adding the equations for $c_{1}$ and $c_{2}$ and integrating over the domain gives

$$
\phi(t) \equiv \frac{\mathrm{d}}{\mathrm{d} t} \int_{\Omega}\left(c_{1}+c_{2}\right) \mathrm{d} x=(a+b)-\int_{\Omega} c_{2} \mathrm{~d} x
$$

where in the quasi-steady state $\phi(t) \approx 0$. The time series $\phi(t)$ for one particular growth function and parameter set is shown in Fig. 3.

Heuristically we may describe the process of generation of a pattern sequence in the model, under the two timescales above, as follows. The initial bifurcation from the homogeneous steady state is through a diffusion-driven instability at a critical point $\gamma=\gamma_{c}$ which may be derived from linear stability theory. Linear growth of the destabilizing mode and subsequent saturation to a large amplitude pattern ensues. The pattern then evolves in two dynamical regimes. The amplitude of the pattern is gently modulated in the quasi-steady state as $\gamma$ changes with time. A pattern persists until at some point the solution undergoes a transition to a new quasi-steady pattern; undergoing a fast dynamic reorganization (activator peak splitting and separation for Schnakenberg kinetics). Subsequently a pattern of higher spatial frequency (for $\gamma$ increasing) is established which in turn persists, with slow amplitude modulation, before the solution undergoes further transitions. 


\section{The FREQUENCY-DOUbLING SEQUENCE AND SELF-SIMILARITY}

Frequency-doubling corresponds to a sequence in which the spatial frequency of the pattern regularly doubles, and no other pattern modes enter the sequence. In dimensional coordinates on the growing domain this corresponds to a regular doubling of the pattern mode. Self-similarity of pattern modes may be used to predict necessary conditions under which this sequence is expected in our model. As $\gamma(t)$ is a monotonically increasing function we can eliminate $t$ in favour of $\gamma$ to give

$$
h(\gamma) \frac{\partial c}{\partial \gamma}=\frac{1}{\gamma} \mathcal{D} \frac{\partial^{2} c}{\partial x^{2}}+R(c) .
$$

Let us assume that at $\gamma=\gamma^{*}$ the solution has spatial profile $c\left(x, \gamma^{*}\right)$. At any point in the sequence, in particular at $\gamma=\gamma^{*}$, a pattern of twice the spatial frequency may be constructed by applying the tent map, given in equation (5). We define $q_{2}(x, \gamma)$ such that

$$
q_{2}\left(x, \gamma^{\prime}\right) \equiv c\left(p_{2}(x), \gamma^{\prime}\right)
$$

which satisfies the evolution equation

$$
h\left(\gamma^{\prime}\right) \frac{\partial q_{2}}{\partial \gamma^{\prime}}=\frac{1}{4 \gamma^{\prime}} \mathcal{D} \frac{\partial^{2} q_{2}}{\partial x^{2}}+R\left(q_{2}\right) .
$$

Returning to the original equation, $c\left(x, 4 \gamma^{\prime}\right)$ satisfies

$$
\frac{1}{4} h\left(4 \gamma^{\prime}\right) \frac{\partial c}{\partial \gamma^{\prime}}=\frac{1}{4 \gamma^{\prime}} \mathcal{D} \frac{\partial^{2} c}{\partial x^{2}}+R(c) .
$$

Now $c\left(x, 4 \gamma^{\prime}\right)$ and $q_{2}\left(x, \gamma^{\prime}\right)$ satisfy the same equation if

$$
h\left(4 \gamma^{\prime}\right)=4 h\left(\gamma^{\prime}\right) .
$$

Recalling that $h(\gamma)=\mathrm{d} \gamma / \mathrm{d} t$, this requires the time dependence of $\gamma$, and hence the domain growth, to be exponential:

$$
r(t)=\exp (\rho t), \quad \gamma(t) \propto \exp (2 \rho t) .
$$

Furthermore, if at $\gamma=\gamma^{*}$ we find that in fact

$$
q_{2}\left(x, \gamma^{*}\right)=c\left(x, 4 \gamma^{*}\right) \quad \forall x \in[0,1],
$$

the matching condition, then the profiles are equivalent and, assuming uniqueness of solutions to the evolution equation for given initial and boundary conditions, they will continue to coincide for all time such that $\gamma>\gamma^{*}$. We have made no 
assumptions about $\gamma^{*}$ or the spatial profile at $\gamma^{*}$; this observation holds in both quasi-steady and fast (transition) dynamical regimes.

We have shown that under exponential growth $q_{2}(x, \gamma)=c\left(p_{2}(x), \gamma\right)$ and $c(x, 4 \gamma)$ satisfy the same evolution equation. Below we investigate the consequences for frequency-doubling. From the spatial profile $c\left(x, \gamma^{*}\right)$ we can construct $q_{2}\left(x, \gamma^{*}\right)=c\left(p_{2}(x), \gamma^{*}\right)$ which by definition has twice the spatial frequency. Let us suppose that for given initial conditions $c(x, \gamma)$ evolves such that for $\gamma \in\left[\gamma^{*}, 4 \gamma^{*}\right]$ the solution is initially mode $m$ and subsequently undergoes a transition to mode $2 m$, such that the match (32) is satisfied. Then the uniqueness of solutions of the evolution equation requires that $q_{2}$ and $c(x, 4 \gamma)$ remain the same for $\gamma>\gamma^{*}$. But by construction $q_{2}$ has twice spatial frequency of $c(x, \gamma)$ and so, in the interval $\gamma \in\left[4 \gamma^{*}, 16 \gamma^{*}\right]$, the solution $c(x, \gamma)$ must consist of the sequence $2 m$ and transition to $4 m$, and so on.

In this way the pattern sequence for $\gamma>4 \gamma^{*}$ may be reduced to the behaviour on $\gamma \in\left[\gamma^{*}, 4 \gamma^{*}\right]$. We now consider this initial interval. In particular, if $c\left(x, \gamma^{*}\right)$ consists of a pattern of mode $m=1$ and we satisfy the matching condition to mode $m=2$ at $\gamma=4 \gamma^{*}$ then our observations suggest that frequency-doubling will naturally ensue in a self-similar cascade. Thus we need only consider the initial stages of the sequence to determine whether frequency-doubling is realized; all subsequent behaviour is equivalent. Furthermore, this analysis predicts that such a sequence generated under exponential growth will undergo frequency-doubling every time $\gamma \rightarrow 4 \gamma$, corresponding to the dimensional domain doubling in length.

Of course we should not require an exact matching (32) and consequently we require some stability properties of the evolution equation. In particular we require that a solution $c(x, \gamma)$ perturbed at some point subsequent to the establishment of a large amplitude pattern remains in the vicinity of the unperturbed solution so that if $c(x, 4 \gamma)$ and $q_{2}(x, \gamma)$ are close at $\gamma=\gamma^{*}$ then they remain close during the interval $\gamma \in\left[\gamma^{*}, 4 \gamma^{*}\right]$. We have performed numerical simulations of the evolution equation and have compared $c(x, 4 \gamma)$ and $q_{2}(x, \gamma)$ numerically, as described below (and see Figs 4 and 5), and conclude that this stability property is demonstrated by the system, at least in some range of the rate-determining parameter $\rho$. If the stability criterion is met then once some pattern is selected by the initial conditions the sequence is fully determined by the dynamics of the evolution equation and the initial conditions play no further part in determining the composition of the sequence. In this sense the patterns contained in the sequence are generated robustly.

\section{NUMERICAL RESUlts For EXPONENTIAL Domain Growth}

We have computed numerical solutions of equation (22) with Schnakenberg kinetics (24), zero flux boundary conditions and exponential growth function (31) using the method of lines for spatial discretisation and Gear's method for integration 


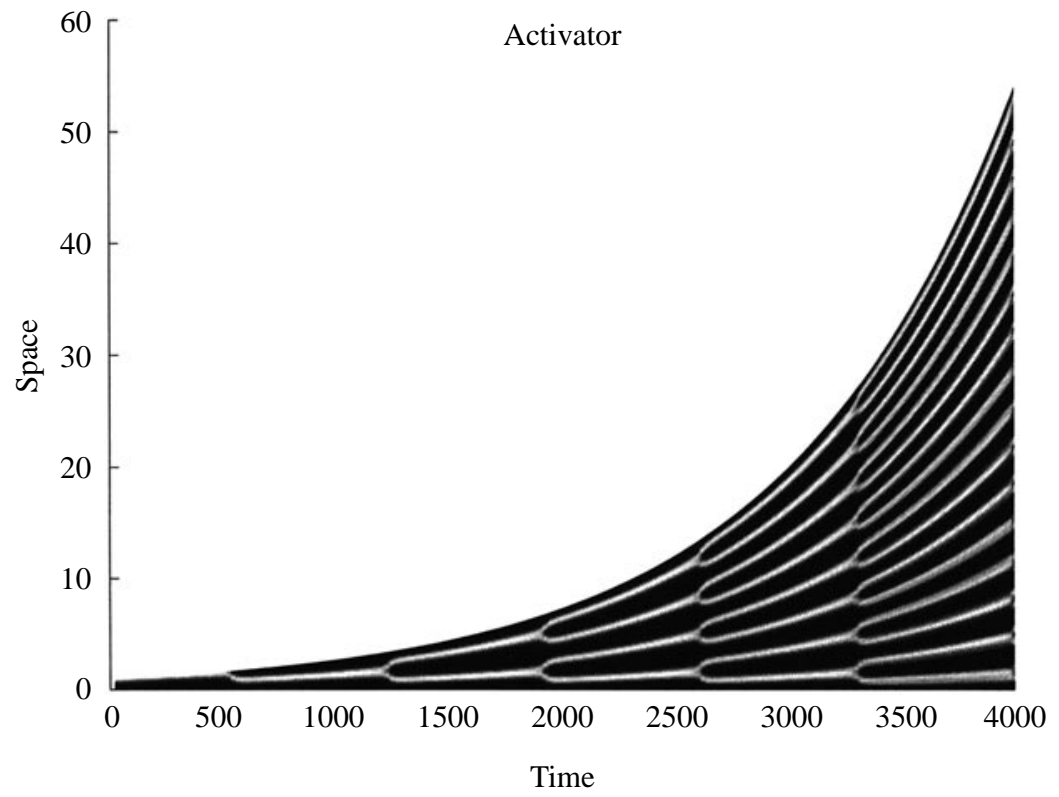

Figure 2. The frequency-doubling sequence. Space-time evolution of activator concentration profile $c_{2}(x, t)$ for Schnakenberg kinetics on the exponentially growing domain with $\rho=0.001$. Light and dark shading represent high and low concentrations respectively. Pattern transitions are by activator peak splitting. The inhibitor profile is in spatial antiphase (the kinetics are of cross type).

in time, as implemented in the NAG numerical routine D03PCF. The kinetic parameters here and in all other simulations with Schnakenberg kinetics are $a=0.1$, $b=0.9$ and we take the ratio of diffusivities $d=0.01$, unless described otherwise. The initial conditions in each case are random fluctuations about the kinetic steady state concentrations, with a uniform distribution and maximum deviation of $0.5 \%$. In all simulations we have at least 10 spatial points per wavelength of pattern. The accuracy in the time integration is $1.0 \times 10^{-6}$. Figure 2 shows a typical solution for the activator species on a growing domain.

Figure 3 shows the evolution of the maximum amplitude $\eta(t)$ for each species (activator and inhibitor) given by

$$
\eta_{i}(t)=\max _{x \in[0,1]}\left(c_{i}(x, t)\right), \quad i=1,2
$$

and $\phi(t)$, defined in equation (25), showing periodic behaviour. The pattern changes each time the domain length doubles corresponding to period $\Delta t=(\ln 2) / \rho$, where $\Delta t \approx 693$ for the parameters in the simulation. The evolution of $\phi(t)$ illustrates the two dynamic regimes, remaining close to zero except during the transition between quasi-steady patterns. 
(a)

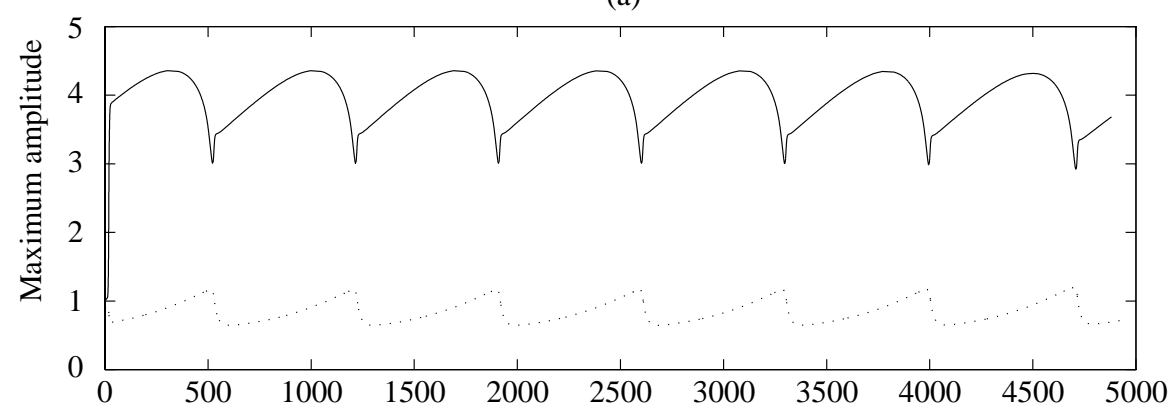

(b)

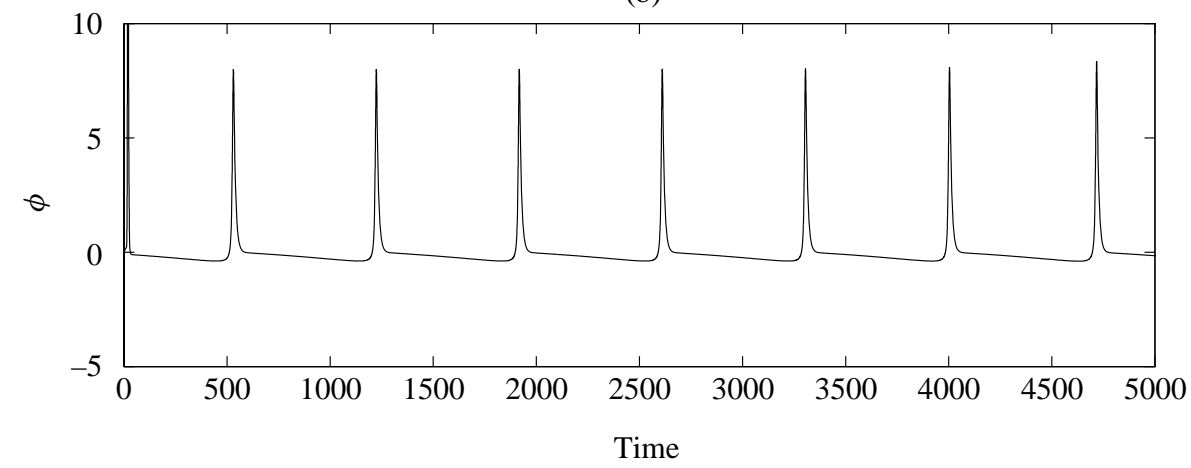

Figure 3. Evolution of (a) maximum pattern amplitude for activator $\eta_{2}(t)$ (solid line) and inhibitor $\eta_{1}(t)$ (dotted line) and (b) $\phi(t)$ for equation (22) with Schnakenberg kinetics and exponential domain growth with $\rho=0.001$.

We have computed $q_{2}(x, \gamma)$ for a solution $c(x, \gamma)$ in order to investigate the matching condition (32) during frequency-doubling. We have implemented a routine which compares the solution at time $t(\gamma)$ with the profile generated by the action of the tent map $p_{2}(x)$ on the solution at time $t(\gamma / 4)$. To do this we use linear interpolation to approximate $c(x, \gamma / 4)$, before applying the tent map to compute $q_{2}(x, \gamma / 4)=c\left(p_{2}(x), \gamma / 4\right)$. In Fig. 4 we show how the maximum deviation over space for the activator depends on the time-step size (which is greater than the deviation for the inhibitor). The maximum occurs during the transition between pattern modes. The time-step is simply the time interval between data records and hence the interval over which we interpolate; it does not reflect the accuracy in time integration. The figure shows that the deviation tends to zero with the step-size. This suggests that we do have $\left|c(x, \gamma)-q_{2}(x, \gamma / 4)\right| \rightarrow 0$ during the transition, and hence the required matching condition. The transition between modes $m=2$ and $m=4$ is illustrated in Fig. 5(a)-(d). Here $c(x, \gamma)$ and $q_{2}(x, \gamma / 4)$ are plotted on the same axes and cannot be distinguished. 


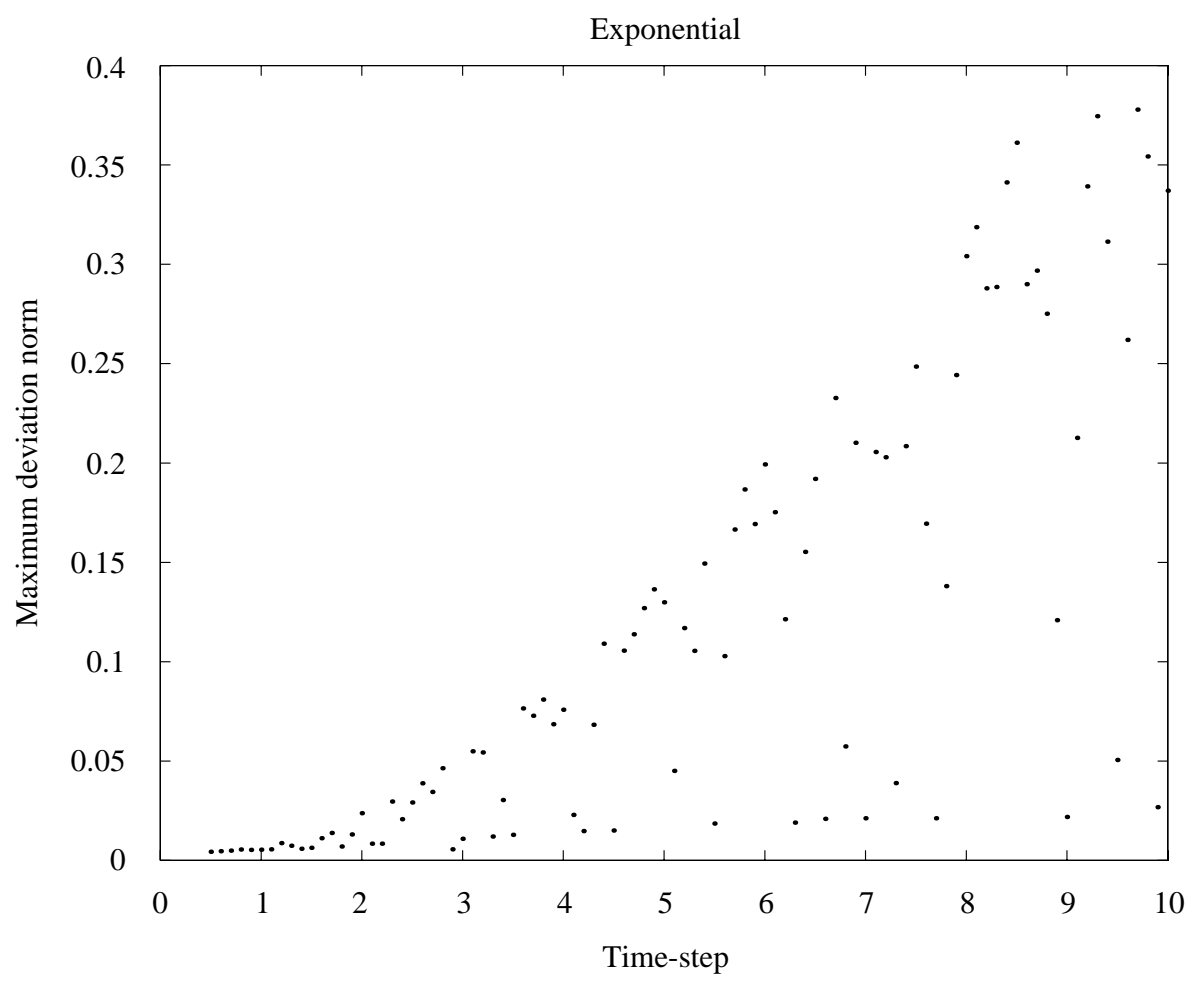

Figure 4. The dependence of $\max _{x \in[0,1]}\left|c(x, \gamma)-q_{2}(x, \gamma / 4)\right|$ during the transition between patterns as a function of the interpolation step size (see text for details). For comparison purposes the activator maximum amplitude $\eta_{2} \approx 3$ during the transition. On closer inspection the data are seen to oscillate. This is an artefact due to the sampling frequency and the sharpness of the transition.

5.1. Dependence on growth rate-determining parameter $\rho$. The frequency-doubling sequence is realized over several orders of magnitude of $\rho$, the growth rate-controlling parameter. Here we examine the pattern sequence under exponential growth at the extremes of the range of validity of $\rho$. Our ability to numerically monitor frequency-doubling into higher modes is severely limited by the mesh capacity that we can obtain computationally. Currently sequences are examined for up to nine frequency-doubling events. We have found that frequency-doubling behaviour under exponential growth with Schnakenberg kinetics is observed over four orders of magnitude in $\rho$ for $d=0.01$; approximately $10^{-6}<\rho<10^{-2}$. The self-similarity argument suggests that for exponential growth the sequence either persists indefinitely or it fails, according to whether the matching condition is achieved, although it gives no insight as to how the failure will occur. Numerically we find that there is a discrete change in behaviour when $\rho$ is decreased through a lower critical value. For $\rho$ below this point, $\rho_{c}$, the pattern sequence does not undergo 
frequency-doubling and different sequences may be obtained for different sets of random initial conditions. Figure 5(i)-(l) illustrate the manner in which this break down occurs during a transition. Here the constraint imposed by the nonautonomy is not sufficient to generate the frequency-doubling sequence. At very small values of $\rho$ correspondingly small time-steps are required numerically to investigate the solution behaviour during the transitions between patterns. Raising $d$ is found also to raise $\rho_{c}$, and in the figure we have taken $d=0.06$ to allow a reasonable time step to be used.

When $\rho$ is large (as $\rho \rightarrow 1$ ) the sequence suffers a gradual break down. Examination of the criterion for the self-similar cascade shows that there is no longer matching for large $\rho$. The peak is not stationary before the transition between modes and subsequently the peak splitting is asymmetrical, as illustrated in Fig. 5(e)-(h). The tent map construction is by definition symmetrical, and here we certainly cannot find a point to satisfy the matching condition (32). In this example the asymmetry arises during the separation of peaks subsequent to peak splitting and the solution undergoes the next transition before reaching a quasi-steady state. The departure from the characteristic alternation between slow and fast dynamical regimes is demonstrated in the behaviour of $\phi(t)$, defined in Section 3.1, as shown in Fig. 6 for three different values of $\rho$. The onset of the asymmetry is gradual and although the solution no longer enters a quasi-stationary state, the number of turning points on the domain continues to double periodically for some further range of $\rho$. When $\rho$ becomes very large, $\rho \sim 1$, the solution is purely transient with no patterns recognizable as quasi-stationary modes.

5.2. Role of the kinetic function. The analysis presented above does not depend critically on the nonlinear part of the kinetic function $R$. Provided that the linearized kinetics permit diffusion-driven instability and the initial matching of solution and construction occurs, then we infer that in general the nonlinearities in the kinetics do not affect the robustness of the sequence. Simulations of the various kinetic models proposed in the literature have produced frequency-doubling sequences with activator peak splitting or insertion. In Fig. 7 we show two such sequences, with peak splitting in the Schnakenberg scheme (24) and peak insertion for the model proposed by Gierer and Meinhardt (1972)

$$
R=\left(\begin{array}{c}
v_{1} c_{2}^{2}-\mu_{1} c_{1} \\
v_{2} c_{2}^{2} / c_{1}-\mu_{2} c_{2}+\delta_{2}
\end{array}\right)
$$

where we take $v_{1}=\mu_{1}=0.02$ and $v_{2}=\mu_{2}=0.01$. In this scheme the kinetics preclude frequency-doubling when $\delta_{2}$ is identically zero by preventing either form of reorganization of the pattern, also shown in Fig. 7. The standard frequencydoubling sequence is recovered for all $\delta_{2} \neq 0$.

It is also possible to generate a sequence in which the pattern undergoes splitting and insertion of new peaks simultaneously, which will be reported elsewhere. 
(a)

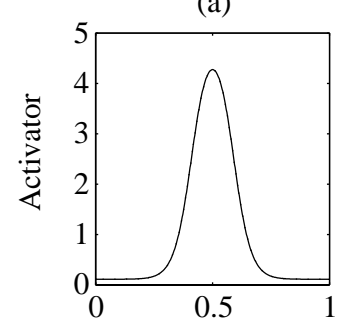

(e)

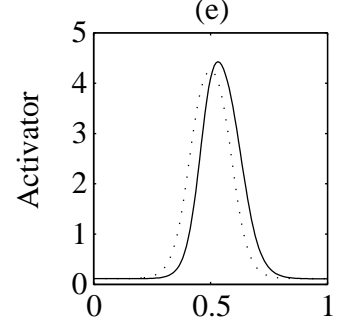

(i)

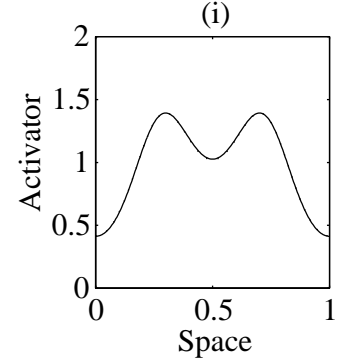

(b)

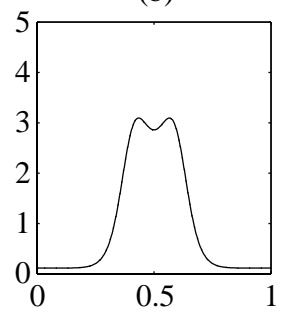

(f)

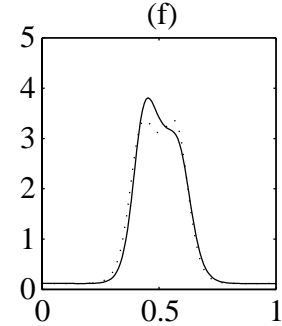

(j)

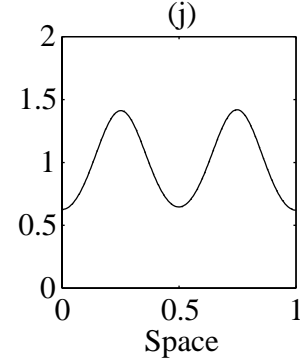

(c)

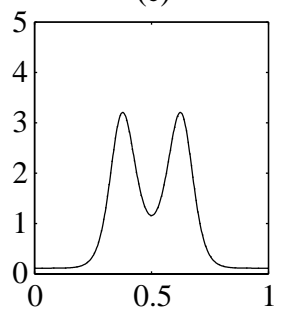

(g)

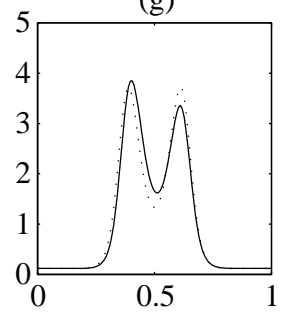

(k)

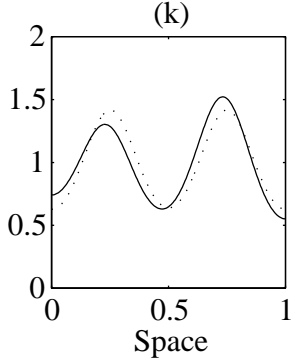

(d)

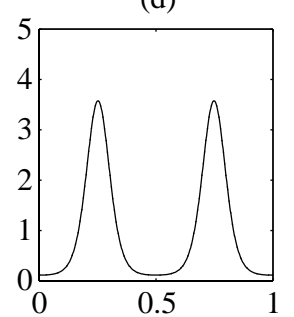

(h)

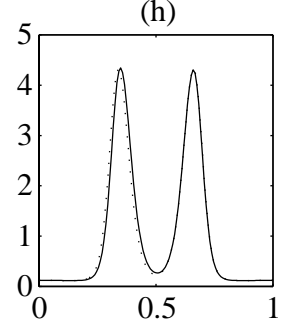

(1)

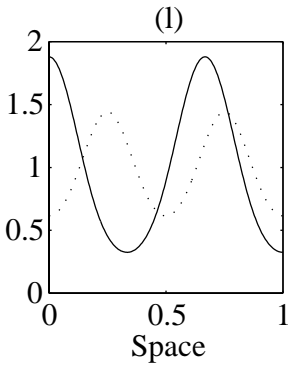

Figure 5. The peak splitting transition between modes $m=2$ and 4 for equation (22) with Schnakenberg kinetics and exponential growth with three different values of $\rho$, the rate-determining parameter, where (a)-(d) $\rho=0.001$, (e)-(h) $\rho=0.02$ where $d=0.01$ and (i)-(l) $\rho=$ $0.00001<\rho_{c}$ with $d=0.06$ (see text for details). The dotted trace shows the (symmetrical) tent map acting on the solution at $\gamma / 4$ [and cannot be distinguished in (a)-(d)].

This behaviour is generic to purely cubic autocatalysis and is structurally unstable to small quadratic terms, the addition of which recovers the frequency-doubling sequence.

\section{Other Growth FUnCtions}

Exponential isotropic growth models a population of cells undergoing cell division at a fixed rate which is independent of spatial or temporal coordinate. While this is a reasonable model of the initial stages of an unconfined growth it is not realistic for many applications. We can use the same analysis to investigate pattern formation under other growth functions. In previous studies various authors 
(a)
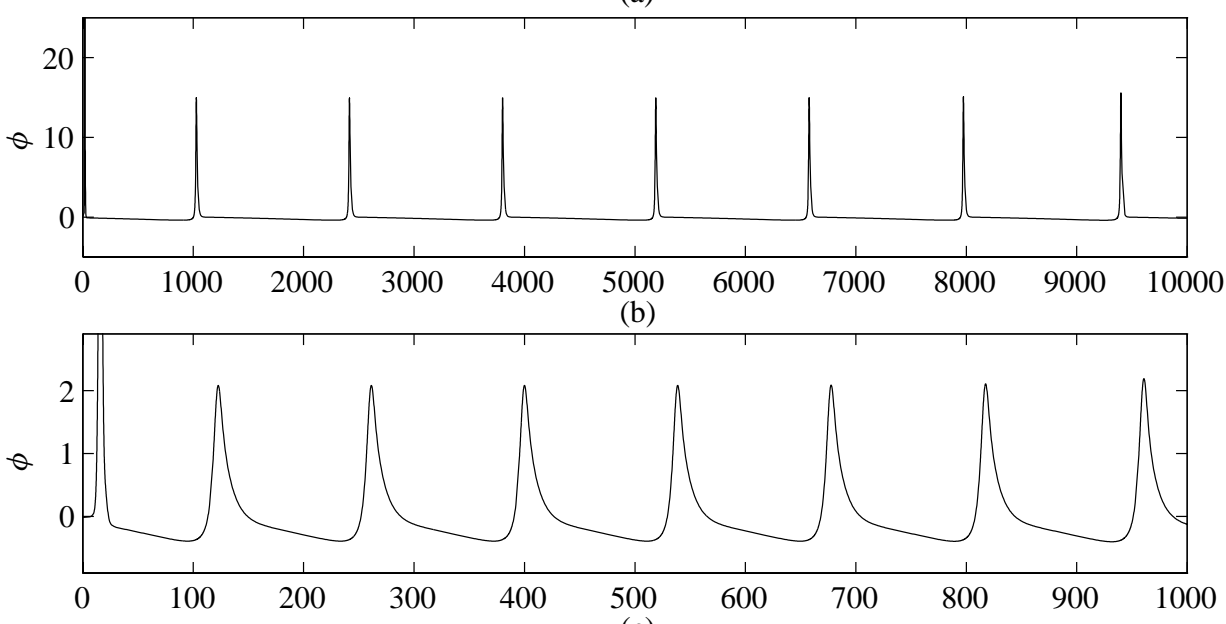

(c)

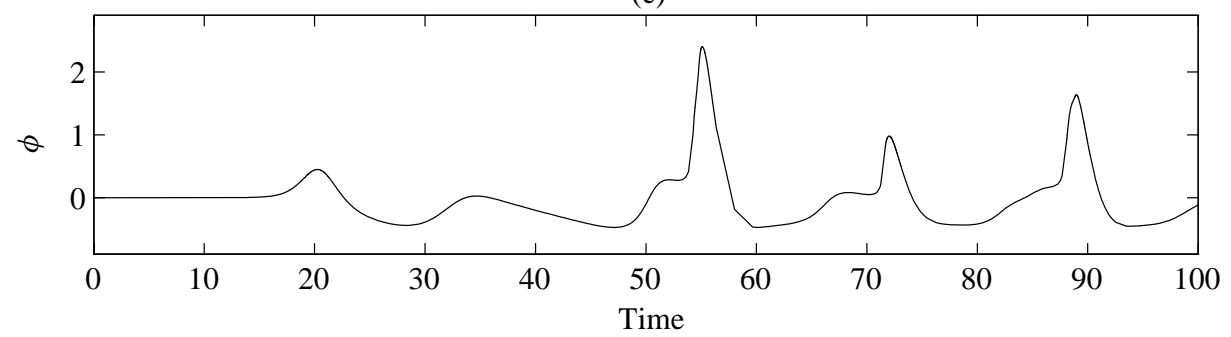

Figure 6. Evolution of $\phi(t)$ for exponential growth with (a) $\rho=0.0005$, (b) $\rho=0.005$ and (c) $\rho=0.05$ showing the departure from two characteristic dynamic regimes.

have considered linear growth. Although this may not have a strong biological motivation, studying this growth function gives further insight into the mechanism of pattern sequence formation. We will also consider the biologically plausible scenario of logistic time dependence, where the final domain size is limited.

6.1. Linear domain growth. For linear growth we have $r(t)=1+\rho t$ giving

$$
\gamma(t)=\gamma_{0}(1+\rho t)^{2}, \quad h(\gamma)=\frac{\mathrm{d} \gamma}{\mathrm{d} t}=2 \rho \sqrt{\gamma_{0} \gamma} .
$$

where for convenience we will take $\gamma_{0}=1$. As before we consider the evolution of a pattern over some interval $[\gamma, 4 \gamma]$ such that on eliminating $t$ the governing equation for $c\left(x, 4 \gamma^{\prime} ; \rho\right)$ is

$$
\rho \sqrt{\gamma^{\prime}} \frac{\partial c}{\partial \gamma^{\prime}}=\frac{1}{4 \gamma^{\prime}} \mathcal{D} \frac{\partial^{2} c}{\partial x^{2}}+R(c) .
$$

Again we consider the frequency-doubled construction, while this time allowing for a change in $\rho$ to find the equivalent equation. Now $q_{2}\left(x, \gamma^{\prime} ; \rho^{\prime}\right)=c\left(p_{2}(x)\right.$, 

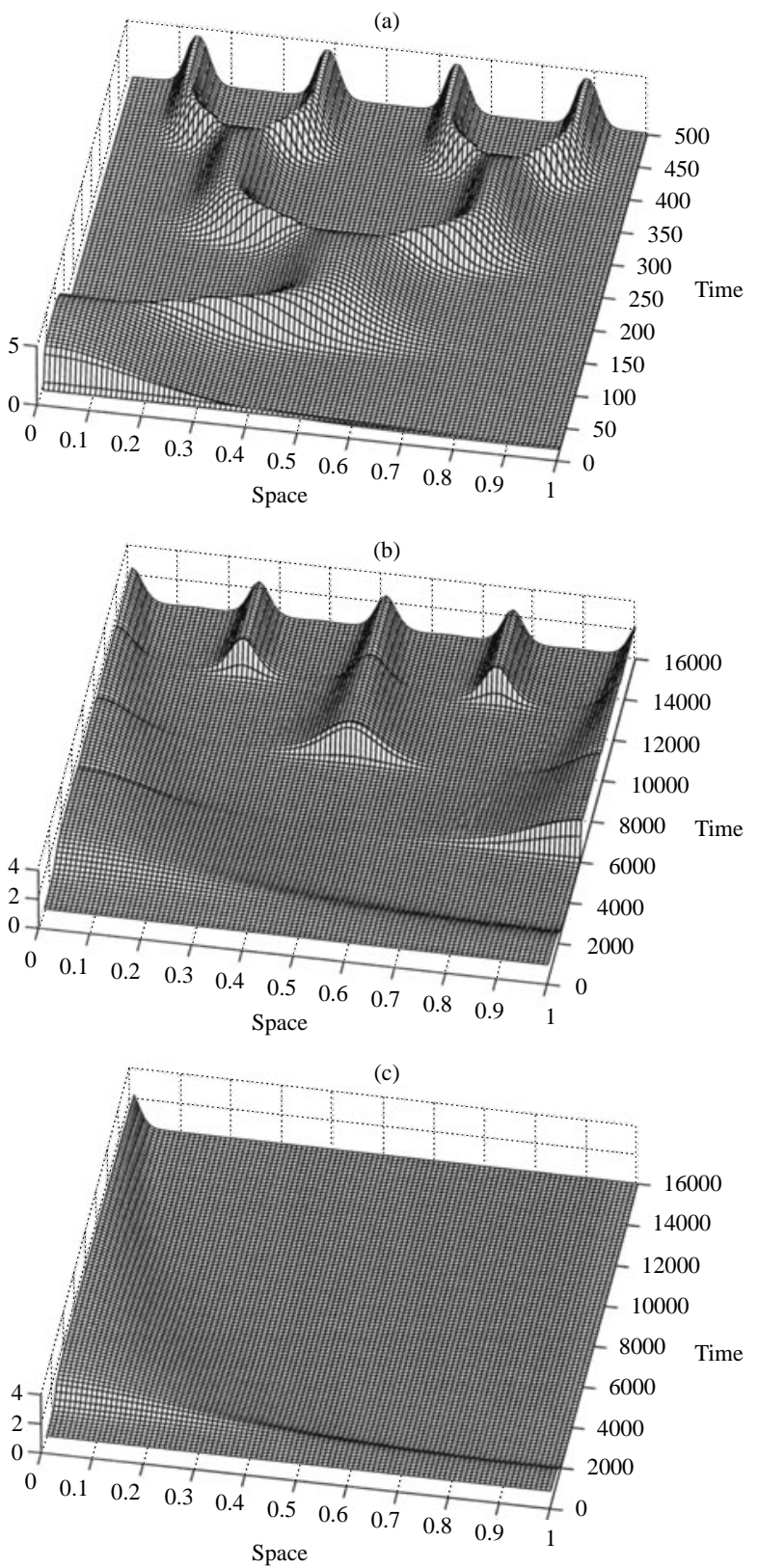

Figure 7. Evolution of the activator species for equation (22). Frequencydoubling with (a) activator peak splitting for Schnakenberg kinetics and (b) peak insertion for the Gierer-Meinhardt model with $\delta_{2}=0.001$, (c) for which $\delta_{2}=0$ precludes pattern reorganization. 
$\left.\gamma^{\prime} ; \rho^{\prime}\right)$ satisfies

$$
2 \rho^{\prime} \sqrt{\gamma^{\prime}} \frac{\partial q_{2}}{\partial \gamma^{\prime}}=\frac{1}{4 \gamma^{\prime}} \mathcal{D} \frac{\partial^{2} q_{2}}{\partial x^{2}}+R\left(q_{2}\right)
$$

so that $q_{2}(x, \gamma ; \rho / 2)$ and $c(x, 4 \gamma ; \rho)$ satisfy the same equation.

The implication of this result is that prolonged frequency-doubling behaviour is not a natural consequence of this growth function. However, as before, if there exists a point $\gamma=\gamma^{*}$ such that

$$
q_{2}\left(x, \gamma^{*} ; \rho / 2\right)=c\left(x, 4 \gamma^{*} ; \rho\right),
$$

then they coincide for all $\gamma>\gamma^{*}$. This implies that if a sequence generated with linear growth rate $\rho$ undergoes $M$ frequency-doubling events before this sequence breaks up, then a sequence generated at growth rate $2 \rho$ must complete $M+1$ such events. Numerical evidence confirming this prediction is presented in Fig. 8. Although the analysis does not give any information about the sequence after the break down of frequency-doubling, numerical solutions suggest that the subsequent behaviour is not robust, and the sequence may be different for each set of initial conditions.

6.2. Comparisons with exponential domain growth. The quasi-steady regime requires that the timescale for pattern formation be sufficiently faster than that for domain growth. We can predict the point of break down of the frequency-doubling sequence under linear growth conditions from knowledge of the lower limit in the rate-determining parameter, $\rho_{c}$, for the sequence under exponential growth. Comparison of growth rates then suggests that frequency-doubling will occur for linear growth when

$$
h_{\text {lin }}(\rho) \geq h_{\exp }\left(\rho_{c}\right)
$$

where $h_{\exp }=2 \rho \gamma$ and $h_{\text {lin }}=2 \rho \sqrt{\gamma \gamma_{0}}$. Then we can derive a condition on $\gamma$ for the break down of the sequence, as illustrated in Fig. 9, namely

$$
\gamma \approx \gamma_{0}\left(\frac{\rho}{\rho_{c}}\right)^{2}
$$

as indicated by the vertical dashed line in the figure.

Numerical simulations have confirmed for linear growth that the point of break down in $\gamma$ varies approximately with the square of $\rho$. From equation (40) we see that higher pattern modes may be admitted prior to the break down of the sequence only if $\rho$ is sufficiently larger than $\rho_{c}$. Thus point of break down observed in a sequence that initially undergoes frequency-doubling, as given by the intersection in the figure, corresponds to time $t^{*}$ such that

$$
t^{*}=\frac{1}{\rho_{c}}-\frac{1}{\rho} \approx \frac{1}{\rho_{c}}
$$



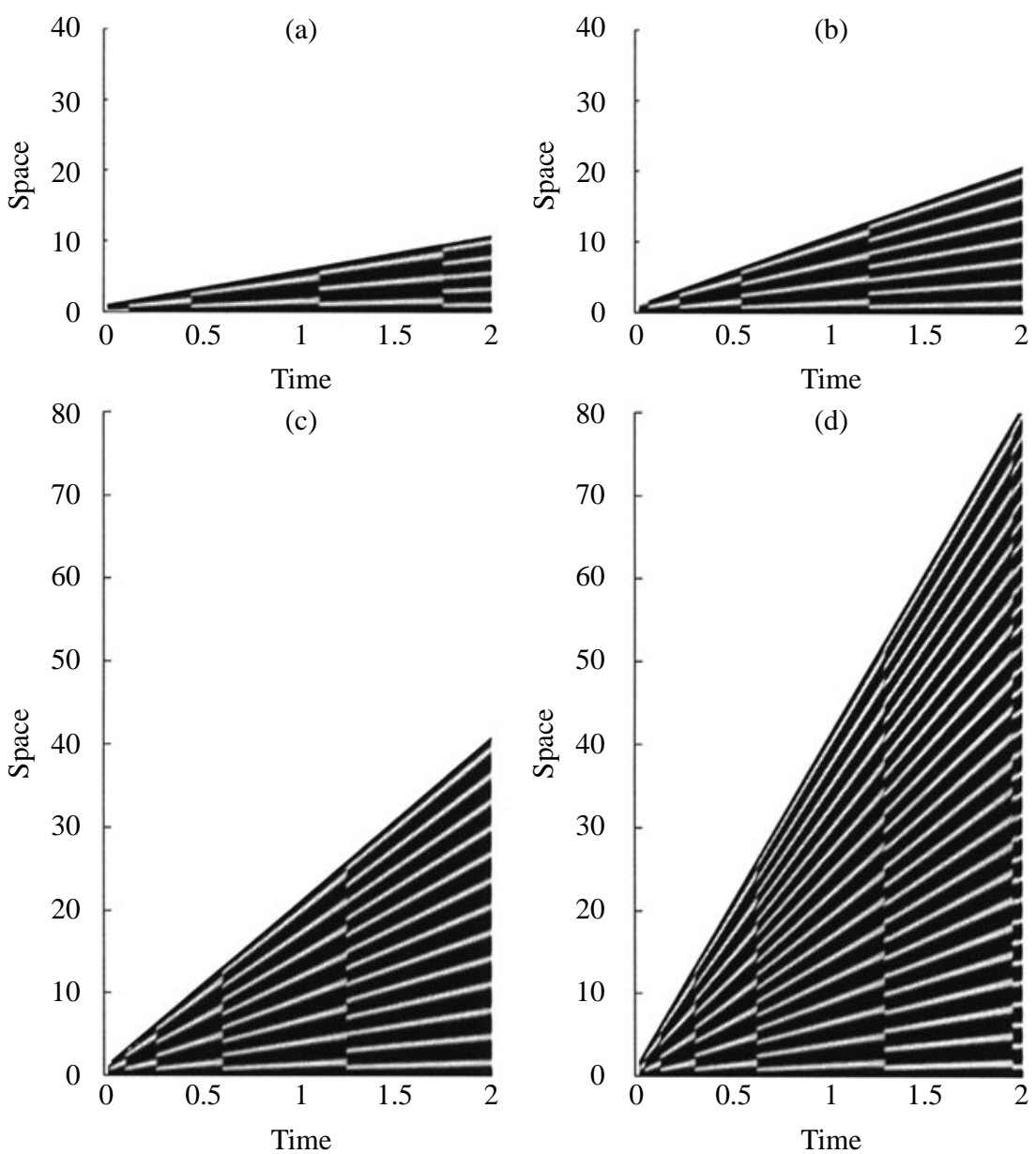

Figure 8. Linear domain growth. Space-time evolution of activator concentration profile $c_{2}(x, t)$ with Schnakenberg kinetics for (a) $\rho=$ $5.0 \times 10^{-6}$, (b) $\rho=1.0 \times 10^{-5}$, (c) $\rho=2.0 \times 10^{-5}$ and (d) $\rho=4.0 \times 10^{-5}$ showing 2, 3, 4 and 5 frequency-doubling transitions respectively before the sequence breaks down. Transitions appear discontinuous because they occur over a time interval much smaller than the total interval shown in the figures. Other simulations have shown that the remaining pattern sequence is nonrobust as to which pattern modes it contains and to when the transitions occur. Time is in units of $10^{6}$. 


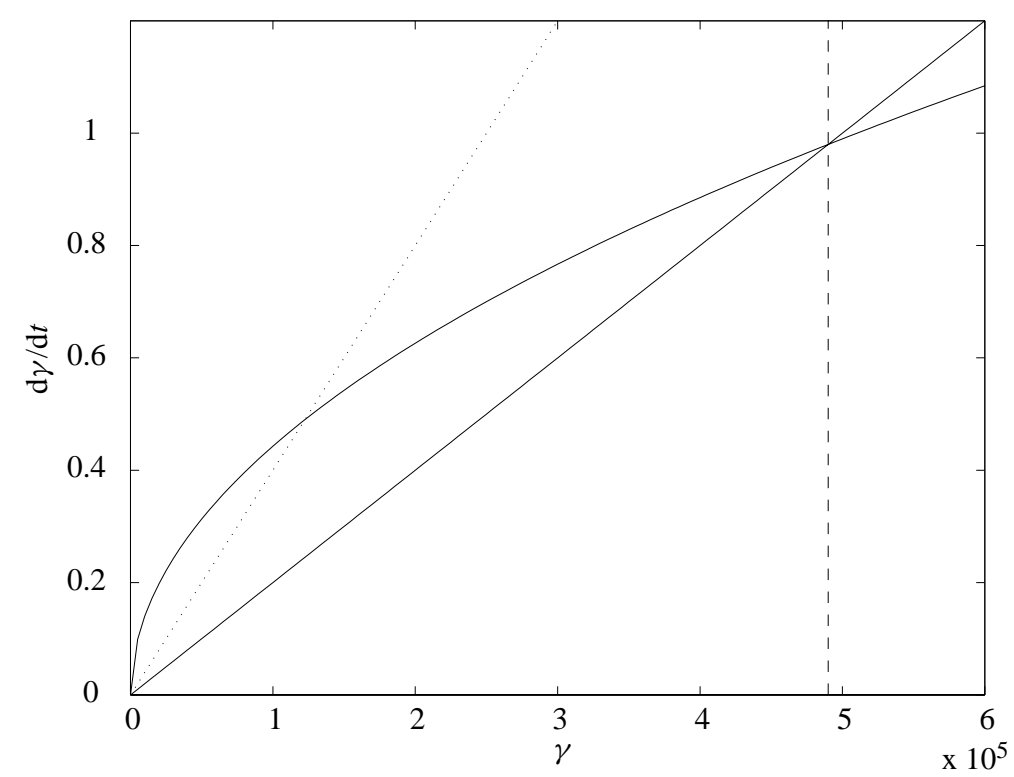

Figure 9. Rate of change of $\gamma$ for exponential (straight line) and linear (curved line) growth functions. The intersection between the linear growth curve and the line for exponential growth at the (lower) critical rate parameter $\rho_{c}$ is marked with a vertical dashed line. The dotted line represents exponential growth for $\rho>\rho_{c}$ for which frequency-doubling is observed.

which, for $\rho_{c} \approx 10^{-6}$, is in agreement with the simulations shown in Fig. 8. In all cases $t^{*}$ falls between the times for the final successful frequency-doubling transition and the subsequent transition which fails to double the spatial frequency.

Analogously to the exponential case, break down of in robustness occurs for $\rho \rightarrow 1$ through the introduction of an asymmetry during transitions between low modes, as the timescales for pattern formation and domain growth coincide.

6.3. Logistic domain growth. If the sensitivity of the tissue to the patterning mechanism is confined to a phase of exponential growth, then the previous results are sufficient to predict the patterning behaviour. However, if pattern is organized during the phase over which the domain growth slows and saturates to achieve some final domain size then we wish to see how this affects pattern formation, and in particular the robustness of the sequence and final pattern that is obtained. Therefore we consider a logistic growth function:

$$
r(t)=\frac{\exp (\rho t)}{1+\frac{1}{\zeta}(\exp (\rho t)-1)}
$$

such that $\mathrm{d} r / \mathrm{d} t=\rho r(1-r / \zeta)$, where $\zeta$ is the ratio of final to initial length.

The logistic growth initially looks exponential, but slows to asymptotically approach the final domain length. If we are in the range for which exponential and 


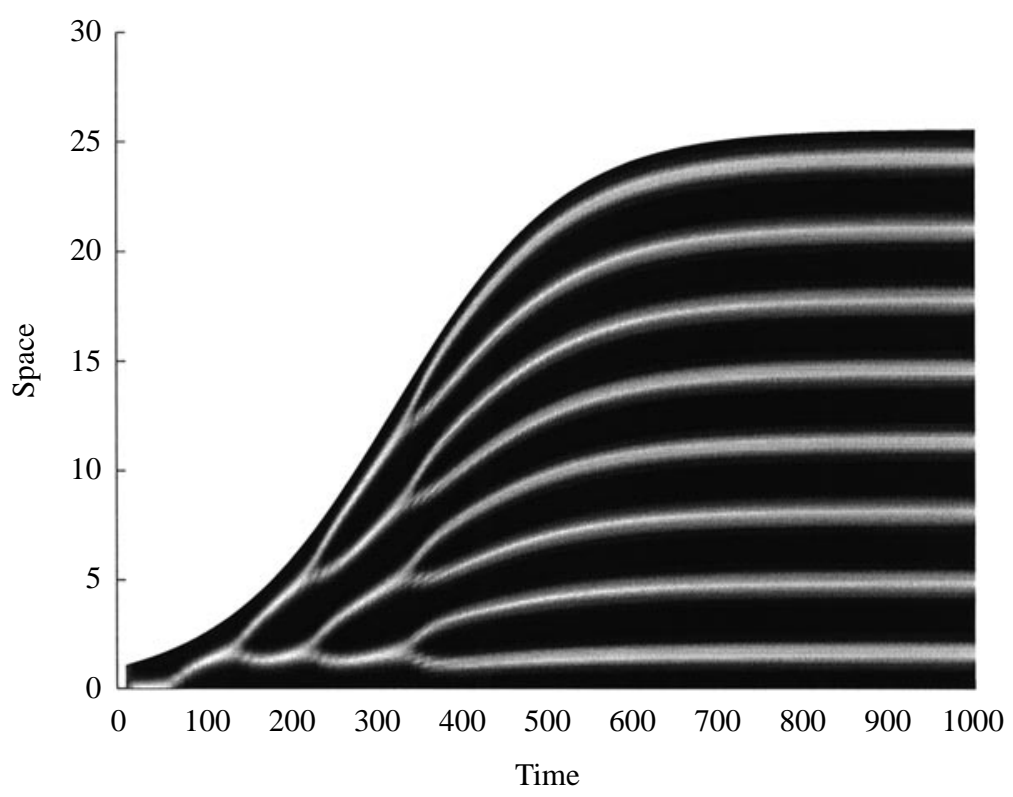

Figure 10. Logistic domain growth. Space-time evolution of activator concentration profile $c_{2}(x, t)$ with Schnakenberg kinetics for logistic growth with $\rho=0.01$ and $\zeta=26.0$. Final robustly generated pattern is mode $m=16$.

linear growth functions give frequency-doubling then we might expect, for a given $\rho$ and for most $\zeta$, that one constituent mode of the frequency-doubling sequence will persist as the domain tends asymptotically to its final length. However, if $\zeta$ is such that the sequence begins to undergo reorganization during the asymptotic approach to final domain size, then nonrobust pattern selection may occur. We will seek to ascertain the relative size of the interval in $\zeta$ which gives such nonrobust behaviour.

In Fig. 10 we show a numerical simulation for logistic domain growth in which mode 16 persists at the final domain size. We have chosen the rate parameter $\rho$ such that the transitions between patterns are seen to be smooth. If, for given $\rho$, a particular mode $m$ of the frequency-doubling sequence persists to the final domain length for the interval $\zeta \in\left[\zeta_{m}^{\min }(\rho), \zeta_{m}^{\max }(\rho)\right]$, then the existence of a window of nonrobust behaviour implies that $\zeta_{2 m}^{\min }>\zeta_{m}^{\max }$. Figure 11 illustrates that the window of nonrobust behaviour exists but is relatively very small. The probability of observing nonrobust final pattern will depend on the relative sizes of $\mathcal{R}_{m}=\zeta_{m}^{\max }-\zeta_{m}^{\min }$ and $\mathcal{N}_{m, 2 m}=\zeta_{2 m}^{\min }-\zeta_{m}^{\max }$, intervals of robust and nonrobust pattern selection respectively, which will depend on $m$ and $\rho$. Numerically we can estimate $\zeta^{\max }$ and $\zeta^{\text {min }}$ for different modes and calculate estimates for $\mathcal{N}_{m, 2 m}$ and $\mathcal{R}_{m}$, and we find that the intervals of nonrobust pattern selection $\mathcal{N}$ are over two orders of magnitude smaller than the intervals, $\mathcal{R}$, for which a robust final pattern 

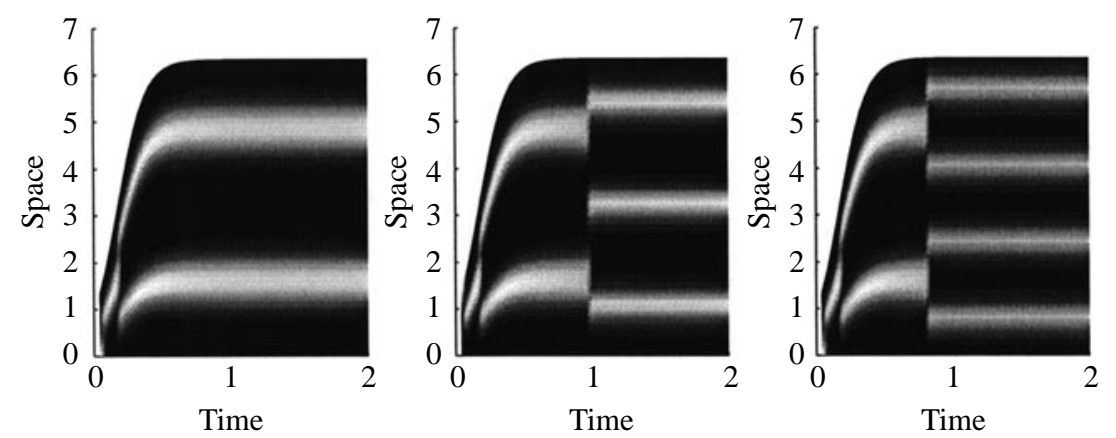

Figure 11. Logistic domain growth: window of nonrobustness. Spacetime evolution of activator concentration profile for Schnakenberg kinetics with logistic growth where $\rho=0.001$ and $\zeta=6.50 \approx \zeta_{4}^{\max }, \zeta=6.51$ and $\zeta=6.52 \approx \zeta_{8}^{\min }$ respectively. Time is in units of $10^{4}$.

from the frequency-doubling sequence is achieved.

\section{Discussion}

In this paper we have considered a model of reaction and diffusion on a slowly and isotropically growing domain which can generate a frequency-doubling sequence of patterns. This sequence is not sensitive to the conditions specified on the initial domain and patterns of fast spatial frequency may be reliably generated. We have predicted with a self-similarity argument that the sequence arises naturally for exponential growth and we have shown numerically that within a range of the exponential growth rate the sequence may continue indefinitely. Under a linear growth function the sequence is found to break down after some number of frequencydoubling events, and a relation between the point of break down and the growth rate is established with the self-similarity argument. For logistic growth one element of the sequence is selected with high reliability, and will persist as the domain tends asymptotically to its maximum size. We have neglected a small contribution to the kinetics, which for exponential growth is a time-independent decay term for each species. It is straightforward to show that the self-similarity analysis is unchanged if the term is included, and we have confirmed numerically that the inclusion of this term makes only small quantitative changes to the solution behaviour.

In the examples presented in this paper we have taken the initial domain length to be below the critical scale for diffusion-driven instability, $\gamma_{0}<\gamma_{c}$. The pattern mode observed after $n$ frequency-doubling events is $2^{n}$. However, other sequences may be generated for initial domain sizes $\gamma_{0}>\gamma_{c}$ such that the first mode to grow is $m_{0}>1$, giving rise to the sequence $m_{0} \times 2^{n}$. If parameters are such that for $m_{0}$ there is no multiplicity of solutions, then a frequency-doubling sequence based on this mode may also be generated by the mechanism we have described. 
We have shown that for the reaction-diffusion model the nonlinearities in the kinetics determine the mechanism of transformation between modes but the frequency-doubling sequence itself is a generic behaviour. We have found a singular behaviour in the Gierer-Meinhardt model where, in the absence of a constant activator production term, the pattern reorganization is prevented. We also report that a class of kinetic schemes with purely cubic nonlinearity undergo reorganization by simultaneous peak splitting and insertion, generating a sequence of frequencytripling. It is straightforward to show that this behaviour is consistent with the selfsimilarity analysis we have presented, where now a matching condition is sought with the solution under the spatial transformation $\bar{p}_{3}(x)$ (see Section 2.3) and as the domain triples in length. It is interesting to note that frequency-doubling and tripling have the same dependence on the nonlinear terms as spot and stripe pattern formation in two spatial dimensions (Ermentrout, 1991).

The pattern sequences capture a degree of insensitivity to domain length, with each pattern element in the sequence persisting as the domain doubles in length. This semi-scale invariance is particularly significant in that it allows regulation, a property of many biological systems, whereby a specific number of pattern elements is laid down despite significant variation in the domain size and without the need to finely tune parameter values. Various authors have considered the problem of scale invariance in reaction-diffusion systems (Othmer and Pate, 1980; Hunding and Sørensen, 1988) and have concluded that some form of feedback from the domain size into the kinetic parameters is necessary, requiring close parameter tolerances. The semi-scale invariance in the present system arises as a natural consequence of the sequence formation mechanism.

7.1. Robust pattern generation. In the context of biological pattern formation, and for reliable pattern formation in noisy environments in general, it is essential that the pattern sequence is not be destroyed by external fluctuations. The frequency-doubling sequence appears to be insensitive to external spatial perturbations imposed as the solution evolves. Simulations in which random perturbations are superimposed at each time-step produce the same sequence. ${ }^{\dagger}$ The transitions between patterns in a sequence are distinct from the initial bifurcation from the homogeneous steady state and associated pattern selection; they are not noise induced and the transient (nonquasi-steady) behaviour appears to be determined entirely by the shape of the evolving solution profile.

We have found that a reaction-diffusion mechanism in one spatial dimension may reliably generate an eight-peak pattern (or 16 , or $32 \ldots$ ). This is contrary to the results of Saunders and Ho (1995) which are based on the numerical analyses of a model by Arcuri and Murray (1986). In the latter paper the authors do not derive a

\footnotetext{
$\dagger$ Integration of a fully stochastic equation is required to fully illustrate this point. We predict that the frequency-doubling sequence on the exponential domain will be insensitive to noise and that the linear domain sequence will be insensitive until the sequence breaks down, with subsequent pattern modes being dependent on the noise and initial conditions.
} 
model from first principles but introduce time dependence to the scaling parameter $\gamma$, which they also use to rescale the time variable: $t=\omega \tau / \gamma$ for dimensional time $\tau$. This transformation distorts the relationship between the timescales for pattern growth and domain growth, and consequently their model fails to produce the robust sequences we have found ${ }^{\ddagger}$. In the present paper we have considered only patterning in one spatial dimension, however, the equations derived earlier describe reaction and diffusion with arbitrary domain growth and in higher dimensions. The extension of the current work into two dimensions is in progress. Preliminary results are encouraging (Painter et al., 1999), in particular that a rectangular domain with preferential growth parallel to the longer axis can sustain frequency-doubling of stripes.

7.2. A universal behaviour? In general, robustness is difficult to obtain for global patterning mechanisms which act simultaneously across a fixed domain. Sequential patterning, such as in the mechanism proposed here on the growing domain, is more reliable. A large family of models of biological pattern formation are based on lateral inhibition and, as discussed by Oster and Murray (1989), have the same underlying mathematical explanation for pattern generation. Whether these other models exhibit qualitatively similar behaviour in response to growth is the subject of current investigation. Murray (1993) and Goodwin et al. (1993) have discussed other scenarios in which the pattern selection problem may be overcome. If patterning is initiated from one sub-region of a domain, then a global pattern may form behind a moving front, generally increasing reliability for the selection of the pattern wavelength. Also it has been shown that if several mechanisms are coupled together in hierarchical systems such that the steady state solution of one mechanism becomes the initial condition, or locally determines parameters for another mechanism, then robustness with respect to initial data may be achieved. This scenario is not unlike the mechanism operating for the growing domain.

\section{ACKNOWLEDGements}

EJC acknowledges an earmarked studentship from the BBSRC. EAG is supported by a Wellcome Trust postdoctoral fellowship. Part of this work was supported by the Institute of Mathematics and its Applications (University of Minnesota) under funds from the National Science Foundation.

\footnotetext{
¥In fact, exploiting the self-similarity argument one can find a time dependence for $\gamma$ such that a frequency-doubling sequence is predicted in Arcuri and Murray's equations, namely $\gamma(t)=\gamma_{0} /(1-$ $\rho t$ ). Numerical simulations support this prediction, however, the entire sequence (as far as can be observed numerically) is confined to the time interval $\left[0, \rho^{-1}\right)$.
} 


\section{REFERENCES}

Arcuri, P. and J. D. Murray (1986). Pattern sensitivity to boundary and initial conditions in reaction-diffusion models. J. Math. Biol. 24, 141-165.

Bard, J. and I. Lauder (1974). How well does Turing's theory of morphogenesis work? J. Theor. Biol. 45, 501-531.

Bentil, D. E. and J. D. Murray (1992). A perturbation analysis of a mechanical model for stable spatial patterning in embryology. J. Nonlin. Sci. 2, 453-480.

Bunow, B., J.P. Kernevez, G. Joly and D. Thomas (1980). Pattern formation by reactiondiffusion instabilities: applications to morphogenesis in Drosophila. J. Theor. Biol. 84, 629-649.

Castets, V., E. Dulos, J. Boissonade and P. De Kepper (1990). Experimental evidence of a sustained Turing-type nonequilibrium chemical pattern. Phys. Rev. Lett. 64, 2953-2956.

Crampin, E. J., E. A. Gaffney, W. W. Hackborn and P. K. Maini. Reaction-diffusion patterns on growing domains: asymmetric growth. (In preparation.)

De Kepper, P., V. Castets, E. Dulos and J. Boissonade (1991). Turing-type chemical patterns in the chlorite-iodide-malonic acid reaction. Physica D 49, 161-169.

Dillon, R., P. K. Maini and H. G. Othmer (1994). Pattern formation in generalized Turing systems I: steady-state patterns in systems with mixed boundary conditions. J. Math. Biol. 32, 345-393.

Ermentrout, B. (1991). Spots or stripes? Nonlinear effects in bifurcation of reactiondiffusion equations on the square. Proc. R. Soc. Lond. A 434, 413-417.

Gierer, A. and H. Meinhardt (1972). A theory of biological pattern formation. Kybernetik 12, 30-39.

Goodwin, B. C., S. Kauffman and J. D. Murray (1993). Is morphogenesis an intrinsically robust process? J. Theor. Biol. 163, 135-144.

Hunding, A. and P. G. Sørensen (1988). Size adaptation in Turing prepatterns. J. Math. Biol. 26, 27-39.

Kondo, S. and R. Asai (1995). A reaction-diffusion wave on the skin of the marine angelfish pomacanthus. Nature 376, 765-768.

Kulesa, P. M., G. C. Cruywagen, S. R. Lubkin, P. K. Maini, J. Sneyd, M. W. J. Ferguson and J. D. Murray (1996). On a model mechanism for the spatial pattering of teeth primordia in the alligator. J. Theor. Biol. 180, 287-296.

Lacalli, T. C., D. A. Wilkinson and L. G. Harrison (1988). Theoretical aspects of stripe formation in relation to Drosophila segmentation. Development 103, 105-113.

Maini, P. K., K. J. Painter and H. N. P. Chau (1997). Spatial pattern formation in chemical and biological systems. J. Chem. Soc., Faraday Trans. 93, 3601-3610.

Murray, J. D. (1981). A pre-pattern formation mechanism for animal coat markings. $J$. Theor. Biol. 88, 161-199.

Murray, J. D. (1982). Parameter space for Turing instability in reaction diffusion mechanisms: a comparison of models. J. Theor. Biol. 98, 143-163.

Murray, J. D. (1993). Mathematical Biology, volume 19 of Biomathematics Texts, 2nd edn, Berlin and London: Springer-Verlag. 
Oster, G. F. and J. D. Murray (1989). Pattern formation models and developmental constraints. J. Exp. Zool. 251, 186-202.

Othmer, H. G. and E. Pate (1980). Scale-invariance in reaction-diffusion models of spatial pattern formation. Proc. Natl. Acad. Sci. USA 77, 4180-4184.

Painter, K. J., P. K. Maini and H. G. Othmer (1999). Stripe formation in Juvenile Pomacanthus explained by a generalized Turing mechanism with chemotaxis. PNAS 96, $5549-5554$.

Saunders, P. T. and M. W. Ho (1995). Reliable segmentation by successive bifurcation. Bull. Math. Biol. 57, 539-556.

Schnakenberg, J. (1979). Simple chemical reaction systems with limit cycle behaviour. $J$. Theor. Biol. 81, 389-400.

Swindale, N. V. (1980). A model for the formation of ocular dominance stripes. Proc. R. Soc. Lond. B 208, 243-264.

Turing, A. M. (1952). The chemical basis of morphogenesis. Phil. Trans. R. Soc. Lond. B 237, 37-72.

Varea, C., J. L. Aragón and R. A. Barrio (1997). Confined Turing patterns in growing systems. Phys. Rev. E 56, 1250-1253. 